\title{
An 8x8 Heterodyne Lens-less OPA Camera
}

\author{
Reza Fatemi, Behrooz Abiri, Ali Hajimiri \\ California Institute of Technology, 1200 E. California Blvd., Pasadena, CA 91125 \\ sfatemi@caltech.edu
}

\begin{abstract}
This paper presents an 8x8 optical phased array (OPA) receiver that operates as a lensless camera using a heterodyne architecture on a thin silicon-photonics integrated SOI substrate. It has a receiving beam width of $0.75^{\circ}$ and beam steering range of $8^{\circ}$.

OCIS codes: (130.0130) Integrated optics; (130.6750) Systems; (110.5100) Phased-array imaging systems;
\end{abstract}

\section{Introduction}

The Integration of photonics components on silicon chips has enabled new applications and novel system designs. Optical phased array transmitter is an example of such integrated systems that has attracted interest in recent years [1] [2]. Proper adjustment of the phase of the light fed to each array element forms a beam of light by adding the output wave of the elements constructively in a certain direction and destructively in other directions. The beam direction is steerable electronically by controlling the phases. Reciprocity of the electromagnetic waves makes it possible to use the same principle for receiving the light coming from a certain direction and rejecting others [3]. This enables a lens-less ultra-thin synthetic aperture OPA camera by sweeping the beam in all directions and putting the measured intensity of the light coming from each direction together and thereby forming an image. In this paper, we present a proof of concept two-dimensional OPA receiver based on heterodyne architecture that operates as an ultra-thin lens-less camera.

\section{Design and Implementation}

The schematic diagram of the phased array receiver camera is shown in Figure 1(a) and the fabricated chip in Figure 1(b). An 8x8 array of grating couplers capture the incident light on the chip surface and each grating coupler guides the light into a waveguide. In this heterodyne scheme, each waveguide is then routed to a directional coupler where the received light is combined with the reference light. The output of the directional coupler is fed to a pair of balanced photodiodes where the signal is mixed down to an electrical intermediate frequency (IF) in the MHz range. The output current of all the photodiodes associated with the receiving elements are summed up by placing them in parallel electrically producing the output signal of the receiver. The reference light is coupled in through a grating coupler and split into 64 paths. Each path goes through a PIN diode phase shifter and feeds a directional coupler. A receive beam is formed by adjusting the phase shifts of each path so that the amplitude of the signal arriving from a certain direction add constructively, while rejecting the intensity of incident light from other directions. This is tantamount to looking in a certain direction.

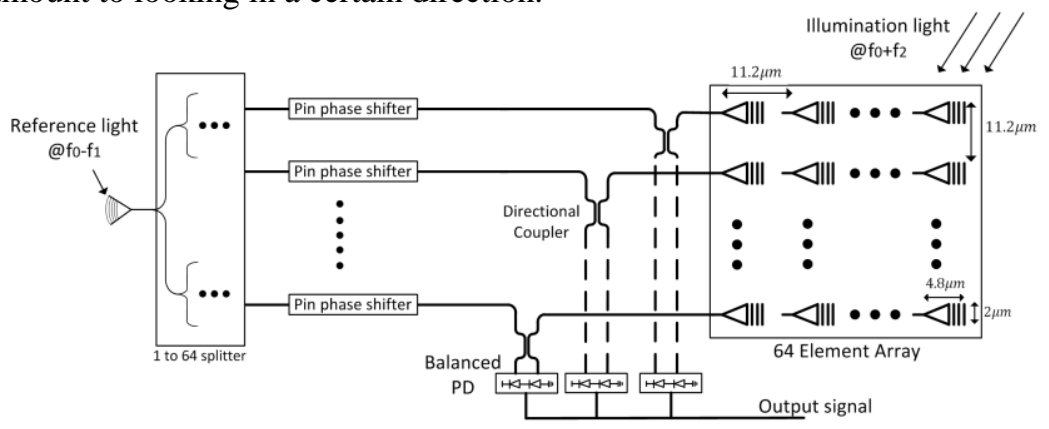

(a)

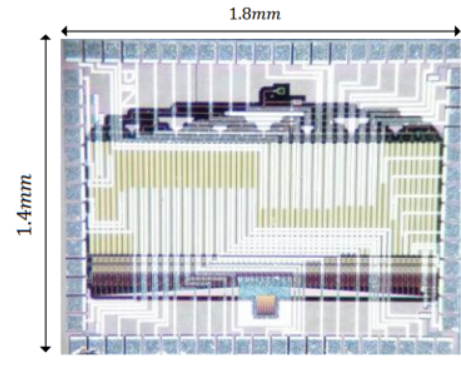

(b)

Figure 1. (a) Schematic of the design (b) Photograph of the chip.

\section{Experimental setup and measurement results}

The chip is mounted on a printed circuit board (PCB) that includes electronic circuit for controlling the phase shifters on the chip and processing the output signal of the chip. A microcontroller is used to control the electronics and communicating with a computer. A $1550 \mathrm{~nm}$ laser is used as the source, whose associated frequency corresponds to $\mathrm{f}_{0}=194 \mathrm{THz}$. The output light of the laser is split into two paths for reference and illumination. Each path is fed to a single-sideband (SSB) modulator. The two SSB modulators shift the optical light frequency in the reference and 
illumination by $\mathrm{f}_{1}=1.15 \mathrm{MHz}$ and $\mathrm{f}_{2}=1.75 \mathrm{MHz}$, respectively. Due to the heterodyne mixing process, the output current of the photodiodes on the chip has the carrier frequency of $f_{I F}=f_{1}+f_{2}=2.9 \mathrm{MHz}$. One advantage of this shifted frequency scheme is that random delay fluctuations in reference and illumination paths (e.g., thermal) manifest themselves as phase noise at the output, not affecting the measured amplitude and therefore not degrading the SNR of the detected signal amplitude. Moreover, 1/f noise of the electronics needed for amplification and processing the output current falls out of band by using a high enough intermediate frequency (IF). A polarization maintaining fiber which carries the reference path is fixed to the chip with transparent adhesive. This allows moving and rotating the receiver for characterization and calibration. In practice, the optical path length of the waveguides has variations because of the fabrication mismatches. An optimization algorithm with random jump search is implemented to calibrate the fabrication mismatches before the imaging phase. The result of optimization provides a lookup table for phase shifter settings to form receiving beams for different azimuth, $\phi$, and elevation angles, $\theta$. Figure 2 shows the measured receiving pattern after calibration for $0^{\circ}$. The beam width is $0.75^{\circ}$ and grating lobes are $8^{\circ}$ apart enabling an 8-pixel by 8-pixel image.

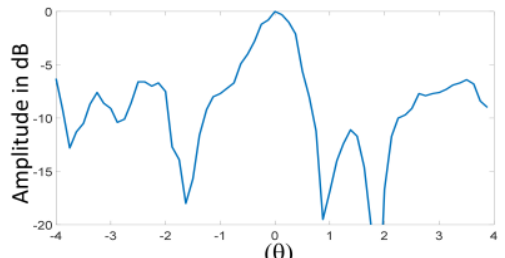

(a)

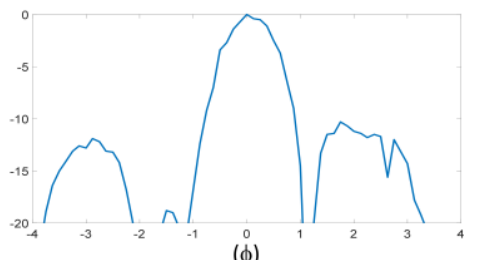

(b)

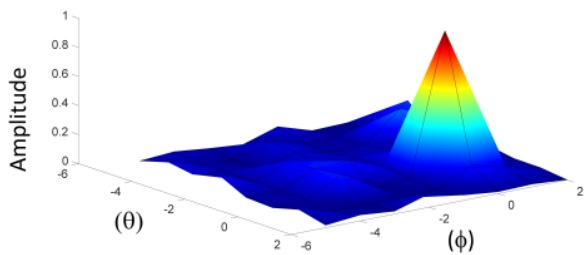

(c)

Figure 2. (a) Normalized receiving pattern cross section of yz-plane (b) Normalized receiving pattern cross section of xy-plane. (c) Normalized 3D pattern.

To illustrate the imaging function of the receiver, a copper tape with a hole is pasted on the plexiglass to block the light except at the hole. The surface of the plexiglass is sanded to enhance scattering, Figure 3(a) and (b). The image of the object is captured by loading the phase setting for different angles and measuring the optical density at each pixel. Figure 3(c) shows the gray scale graph of the measurement result. The white bright spot clearly shows the position of the hole.

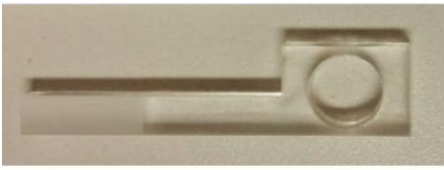

(a)

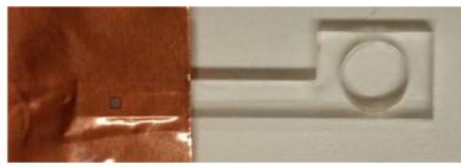

(b)

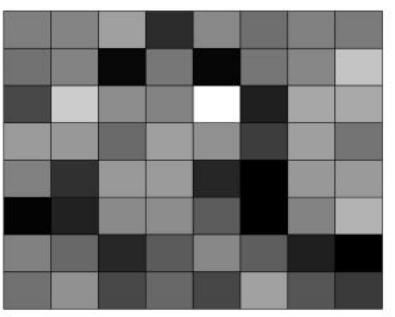

(c)

Figure 3. (a) Sanded plexiglass (b) Copper tape covered imaging target. (c) Captured image in gray scale

\section{Conclusions}

A 2D lens-less ultra-thin camera based on optical phased array receiver is demonstrated. The camera has the beam width of $0.75^{\circ}$ and $8^{\circ}$ of field of view. The beam is electronically steerable for imaging.

\section{References}

[1] B. Abiri, F. Aflatouni, A. Rekhi, A. Hajimiri, "Electronic two-dimensional beam steering for integrated optical phased arrays," in Optical Fiber Communications Conference and Exhibition (OFC), 2014, vol., no., pp.1-3, 9-13 March 2014.

[2] H. Abediasl and H. Hashemi, "Monolithic optical phased-array transceiver in a standard SOI CMOS process," Opt. Express 23, 6509-6519 (2015).

[3] R. Fatemi, B. Abiri, and A. Hajimiri, "A one-dimensional heterodyne lens-Free OPA camera," in Conference on Lasers and Electro-Optics, OSA Technical Digest (2016) (Optical Society of America, 2016), paper STu3G.3. 